\title{
The retention of health human resources in primary healthcare centers in Lebanon: a national survey
}

\author{
Mohamad Alameddine ${ }^{1}$, Shadi Saleh ${ }^{2}$, Fadi El-Jardali ${ }^{2 *}$, Hani Dimassi $^{3}$ and Yara Mourad ${ }^{4}$
}

\begin{abstract}
Background: Critical shortages of health human resources (HHR), associated with high turnover rates, have been a concern in many countries around the globe. Of particular interest is the effect of such a trend on the primary healthcare (PHC) sector; considered a cornerstone in any effective healthcare system. This study is a rare attempt to investigate PHC HHR work characteristics, level of burnout and likelihood to quit as well as the factors significantly associated with staff retention at PHC centers in Lebanon.

Methods: A cross-sectional design was utilized to survey all health providers at 81 PHC centers dispersed in all districts of Lebanon. The questionnaire consisted of four sections: socio-demographic/ professional background, organizational/institutional characteristics, likelihood to quit and level of professional burnout (using the Maslach-Burnout Inventory). A total of 755 providers completed the questionnaire (60.5\% response rate). Bivariate analyses and multinomial logistic regression were used to determine factors associated with likelihood to quit.

Results: Two out of five respondents indicated likelihood to quit their jobs within the next 1-3 years and an additional $13.4 \%$ were not sure about quitting. The top three reasons behind likelihood to quit were poor salary (54.4\%), better job opportunities outside the country (35.1\%) and lack of professional development (33.7\%). A U-shaped relationship was observed between age and likelihood to quit. Regression analysis revealed that high levels of burnout, lower level of education and low tenure were all associated with increased likelihood to quit.

Conclusions: The study findings reflect an unstable workforce and are not conducive to supporting an expanded role for PHC in the Lebanese healthcare system. While strategies aiming at improving staff retention would be important to develop and implement for all PHC HHR; targeted retention initiatives should focus on the young-new recruits and allied health professionals. Particular attention should be dedicated to enhancing providers' role satisfaction and sense of job security. Such initiatives are of pivotal importance to stabilize the workforce and ensure its longevity.
\end{abstract}

Keywords: Retention, Human resources, Primary healthcare, Professional burnout, Lebanon

\section{Introduction}

Thirty years after the Alma-Ata Declaration, which officially launched Primary Healthcare (PHC) in 1978, PHC remains high on the international agenda. It was the theme of the World Health Report in 2008 entitled "Primary Health Care: now more than ever" [1]. This renewed commitment to PHC stems from the universal belief that $\mathrm{PHC}$ is the

\footnotetext{
* Correspondence: fe08@aub.edu.lb

${ }^{2}$ Associate Professor, Faculty of Health Sciences, Department of Health

Management and Policy, American University of Beirut, Riad El-Solh, Beirut 1107

2020, Lebanon

Full list of author information is available at the end of the article
}

most effective and efficient approach to maintain population health and prevent disease progression [2-4]. Yet, the success of these renewed attempts to rejuvenate PHC services is dependent on adequate and well-qualified health human resources (HHR) and the presence of evidencebased retention strategies [5]. Although the role of PHC has lately been expanding in many countries, human resource management has not been awarded the same wellmerited attention [6]. 
This study surveys PHC HHR to explore factors significantly associated with their retention in PHC centers in Lebanon, a subject not yet systematically investigated.

\section{Background}

\section{The global shortage of health human resources}

Being the crucial core of any health system, the presence of an adequate number of well trained and properly experienced HHR is central to the delivery of patientcentered healthcare services across the continuum of healthcare [5,7]. HHR literature concurs in reporting critical shortages of health workers in many countries around the globe, particularly shortages in physicians and nurses [8-10]. Such shortages are confirmed within the context of the Eastern Mediterranean Region (EMR), particularly in low and middle income countries [11].

Most medical interventions require the services of doctors, nurses and other health professionals. Their performance is the first determinant of the quality, efficacy, efficiency, accessibility and viability of health services [2,7].Studies that examined the hospital context established a link between HHR densities and health outcomes; including quality of care, morbidity and mortality $[7,12]$. Furthermore, HHR account for a high proportion of budgets assigned to the health sector, and health expenditures are strongly linked to the ways in which HHR are deployed and used. Finally, the competences and availability of an appropriate workforce is a critical prerequisite for the growth and development of any organization [5]. Still, HHR has been largely a neglected component of the health-system development; in best cases not given the due importance that they deserve.

\section{The retention of the primary healthcare workforce}

Literature over the past two decades has examined the escalating PHC HHR crisis, elaborating on the centrality of recruitment and retention of PHC HHR for building balanced, effective and sustainable primary care services [9,13-15]. Moreover, the role of HHR has been acknowledged to be even more important in community-based care, which tends to use less advanced equipment and is more dependent on competent personnel [5].

The most well documented cause of turnover among health professionals is related to job dissatisfaction [16,17], to which various aspects are attributed. Literature presents a plethora of such factors that influence the retention of HHR; they could be summarized under the following three main categories: organizational characteristics, work characteristics, and individual characteristics. Organizational factors documented include salaries and benefits [18,19], along with organizational commitment and managerial support [20,21]. Work characteristics revolved around the nature of the job, which includes the workload, work en- vironment, and work group cohesion $[17,20]$ as well as opportunities for professional development $[18,19,22]$. Individual socio-demographic characteristics associated with turnover included age, education, professional position, and tenure $[18,20,23]$.

\section{The primary healthcare context in Lebanon}

The first call to the building of a Lebanese PHC system goes back to 1972 [24]. Since then, significant progress has been witnessed on both the public and private fronts as evident by the proliferation and expansion of $\mathrm{PHC}$ facilities across the Lebanese territories. On the public front, the Lebanese Ministry of Public Health led the establishment of a national PHC network that includes 140 centers [25]. The Ministry also led multiple initiatives to strengthen the PHC system, including: partnership for delivery with municipalities and private centers, the development of guidelines and health education materials, distribution of vaccines, drugs, supplies and equipment, as well as training activities [25].

Furthermore, the last few years witnessed the strengthening of privately owned and operated networks of PHC center. This astonishingly fast growth made private $\mathrm{PHC}$ centers the major provider of PHC services in Lebanon and has motivated the public sector to contract with a number of private centers for the provision of publicly funded PHC.

Although increased interest in the PHC sector in Lebanon has instigated the writing of a number of research reports over the last two decades [26,27], none have systematically examined factors significantly associated with retention in this vital sector.

This study aims at investigating PHC health providers' work characteristics, level of burnout and likelihood to quit and identify the factors that are significantly associated with staff retention at PHC centers in Lebanon. To our knowledge, this is the first study to address this subject in Lebanon and the EMR.

\section{Methods}

\section{Research design}

A cross-sectional design was utilized to survey all health providers in $81 \mathrm{PHC}$ centers dispersed in all districts and governorates of Lebanon.

\section{The setting and participants}

Six major private networks providing primary healthcare services in Lebanon were identified and invited to participate in this study. Four agreed to participate; the other two did not for administrative and logistic purposes. The four participating networks have a large beneficiary base, running a total of 81 PHC centers dispersed nationally in all 26 districts of Lebanon. These networks are estimated to employ close to half of the 
primary healthcare providers working in the private sector in Lebanon.

These PHC centers offer health maintenance, promotion and prevention services, as well as a wide range of curative health services including access to generalists, specialists, diagnostic and dental services in various Lebanese areas with service coverage in both rural and urban settings. Although the health promotion and disease prevention services have been expanding over the last few years, it is noteworthy to mention that the focus of PHC centers services in Lebanon has been on delivery of curative and diagnostic services. Furthermore, active public participation remains deficient and indeed needs to be strengthened in the near future.

The target population within these centers was all health providers including: general practitioners, medical specialists (cardiologists, gynecologists, pediatricians, general surgeons and others), nurses, dentists, laboratory and radiation technologists, nutritionists and providers from others specialties (dental assistants, midwives, etc.). In each PHC center, all providers with a minimum of six months of cumulative work experience in the PHC setting were invited to fill the questionnaire. Non-providers, those involved in the management and administration of the PHC centers, were excluded.

Public PHC centers were excluded from this study for several reasons, mainly due to private $\mathrm{PHC}$ centers providing the majority of PHC services in Lebanon [26] and the minimal variability in recruitment and retention policies across public PHC centers. Indeed, there has been a freeze on HHR recruitment and very little turnover with respect to current staff at public PHC centers, whereby all staff are salaried governmental employees. In contrast, evidence shows variability across private PHC centers with respect to recruitment and retention policies.

\section{Ethical approval}

The study protocol, data collection instruments and consent forms were reviewed and received approval by the Social and Behavioral Sciences Institutional Review Board at the American University of Beirut. Written consent was obtained from all respondents, by ticking on the 'I agree' box on the cover page of the questionnaire.

\section{The survey instrument}

Data was collected through a questionnaire consisting of four sections: (1) socio-demographic and professional background, (2) organizational and institutional characteristics, (3) likelihood to quit, and (4) level of burnout using the Maslach Burnout Inventory (MBI). The questionnaire was reviewed and approved for content validity by a multi-disciplinary expert panel consisting of a health management and policy researcher, a clinician, nurses and a statistician. The questionnaire was translated to Arabic then back translated to English and compared to the original. No major differences were found. All language versions of the questionnaire were pilot-tested on $40 \mathrm{PHC}$ providers (excluded from the study) to check for clarity of questions and to ensure that all aspects intended to be measured were covered. No major changes were made.

The dependent variable in this study, likelihood to quit, was measured by asking surveyed PHC providers to answer the following question "How likely are you to quit your current job in the next 1-3 years". Responses were measured on a five-point Likert scale with the following options: Very unlikely, unlikely, not sure, likely, very likely. This measure of likelihood/intention to quit is well documented in literature [21,28-31].

\section{The maslach burnout inventory}

The MBI is the most frequently used instrument to measure occupational burnout. It is a 22 -item self-assessment tool that measures burnout syndrome across three subscales: emotional exhaustion-EE (9 questions), depersonalization-DP (5 questions) and personal accomplishment-PA (8 questions). The EE subscale measures workers' feelings of emotional weariness from one's work. The DP subscale measures workers' impersonal manner in responding to recipients of services. The subscale of PA measures workers' feelings of professional achievement and experience of intrinsic values at work.

The questions were answered according to a sevenpoint frequency scale ranging from "Never" (given a score of zero) to "Daily" (given a score of six). The scores for the questions related to each of the three subscales were added to compute a subscale score, which was then compared to the provided cut-off points for medical professions to establish the corresponding level of burnout [32].

\section{Data collection}

The principal investigators met with the general administration of the four PHC networks in order to explain the objectives of the study. Upon obtaining approval of participation, managers at the selected PHC centers were asked to distribute the questionnaires to all providers fitting the inclusion criteria. Completion of the questionnaire was voluntary. Anonymity of respondents was maintained, with no personal identifiers used and questionnaires returned in sealed envelopes. A total of 755 out of 1247 PHC providers completed the questionnaire (response rate $60.5 \%$ ).

\section{Data analysis}

Upon completion of data collection, a random sample of $10 \%$ of the questionnaires was checked for completeness and accuracy to guarantee the quality and integrity of 
Table 1 Demographic and professional characteristics of survey respondents $(n=755)$

\begin{tabular}{ll}
\hline Variable & $\mathbf{N}(\%)$ \\
\hline Gender & \\
Male & $374(49.6 \%)$ \\
Female & $380(50.3 \%)$ \\
Missing & $1(0.1 \%)$ \\
\hline Age group & \\
18-25 & $84(11.1 \%)$ \\
$26-35$ & $226(29.9 \%)$ \\
$36-45$ & $207(27.4 \%)$ \\
$46-55$ & $185(24.5 \%)$ \\
$>55$ & $45(6.0 \%)$ \\
Missing & $8(1.1 \%)$ \\
\hline Marital status & \\
Single & $223(29.5 \%)$ \\
Married & $491(65.0 \%)$ \\
Others & $39(5.2 \%)$ \\
Missing & $2(0.3 \%)$ \\
\hline Education
\end{tabular}

\begin{tabular}{ll}
\hline Education & \\
Intermediate/High School & $15(2.0 \%)$ \\
Vocational or diploma & $95(12.7 \%)$ \\
Nursing Degree & $209(27.9 \%)$ \\
University Degree & $109(14.5 \%)$ \\
Medical Degree & $135(18.0 \%)$ \\
Medical Specialty & $187(24.9 \%)$ \\
\hline Current position in PHC & \\
Generalist (includes dentists) & $174(23.0 \%)$ \\
Medical Specialist & $164(21.7 \%)$ \\
Nurse & $247(32.7 \%)$ \\
Allied Health Professional & $114(15.1 \%)$ \\
Other health professional & $56(7.4 \%)$
\end{tabular}

\begin{tabular}{ll}
\hline Number of years spent working in PHC & \\
Six months to 1 year & $93(12.3 \%)$ \\
1 to 5 years & $271(35.9 \%)$ \\
6 to 10 years & $170(22.5 \%)$ \\
More than 10 years & $212(28.1 \%)$ \\
Missing & $9(1.2 \%)$ \\
\hline
\end{tabular}

Number of years working in current PHC center

Six months to 1 year

$136(18.0 \%)$

1 to 5 years

$331(43.8 \%)$

6 to 10 years

$145(19.2 \%)$

More than 10 years

$121(16.1 \%)$

Missing

$22(2.9 \%)$

Employment status at PHC

Permanent- Full time

$428(56.7 \%)$

Permanent- Part time
Table 1 Demographic and professional characteristics of survey respondents $(\mathbf{n}=\mathbf{7 5 5})$ (Continued)

\begin{tabular}{ll}
\hline Temporary/Casual & $62(8.2 \%)$ \\
Missing & $9(1.2 \%)$ \\
\hline $\begin{array}{l}\text { One-way travel time to work } \\
\text { Less than } 15 \text { minutes }\end{array}$ & $304(40.3 \%)$ \\
15- 30 minutes & $273(36.2 \%)$ \\
More than 30 minutes & $161(21.3 \%)$ \\
Missing & $17(2.2 \%)$ \\
\hline
\end{tabular}

the collected data. Full data was then exported to and analyzed using the Statistical Package for the Social Sciences (SPSS), version 19.0. Sample characteristics were summarized using frequency and percentages. Likelihood to quit was the outcome variable of interest, and a multinomial logistic regression model was used to explain factors associated with the likelihood to quit. All analyses were conducted at a 0.05 significance level.

\section{Results}

\section{Sample description}

The demographic, socio-economic and professional characteristics of the respondents are shown in Table 1. The gender distribution of the sample was almost half male $(49.6 \%)$ and half female $(50.3 \%)$. The majority of the respondents $(29.9 \%)$ fell in the relatively young age group of 26 to 35 years of age, and nearly two thirds (65.0\%) were married.

Nurses were the largest professional group surveyed at PHC centers (32.7\%), followed by generalists (23.0\%) and medical specialists (21.7\%). More than a third of surveyed providers $(35.9 \%)$ had been working in the PHC setting for one to five years. Over half of the respondents (56.7\%) were working full-time at the $\mathrm{PHC}$ centers and an additional third were working on a parttime basis. Two fifth of them (40.3\%) reported less than 15 minutes one-way travel time to work.

\section{Likelihood to quit}

Likelihood to quit within the next 1-3 years was investigated among providers at PHC centers; close to two fifth of the respondents (39.4\%) indicated likelihood to quit their jobs (likely or very likely) and an additional 13.4\% were not sure about staying or quitting (Table 2).

Surveyed providers gave a variety of reasons behind willingness to quit their job, the top five of which were: poor salary (54.4\%), better job opportunities outside the country (35.1\%), lack of professional development (33.7\%), job instability (31.6\%) and the lack of support from the administration (31.2\%). Moreover, when asked about the reasons behind their choice to work in the current PHC center, the following reasons were the most commonly cited by 
respondents: the nature of the job, potential benefits to the family, proximity to household and area of work.

Also displayed in Table 2 are the findings related to the three subscales of burnout. Analysis reveals that experience of high burnout among providers is related to EE in almost a quarter of the respondents, DP in $13.8 \%$ of respondents, and PA in $18.7 \%$ of the respondents.

\section{Bivariate analyses with likelihood to quit}

Table 3 reports bivariate associations between characteristics of providers and likelihood to quit. Higher burnout levels on all three subscales [EE (p-value<0.001), PA ( $\mathrm{p}$-value $=0.010)$, and DP ( $\mathrm{p}$-value $=0.002)]$ were significantly associated with likelihood to quit.

Likelihood to quit differed by level of education ( $p$-value $=0.045)$ and number of years since graduation (p-value $<0.001)$. The pattern of correlation was such that providers with more specialized education and more years of work experience were less likely to quit. The number of years working in PHC was also shown to have a significant association with likelihood to quit ( $\mathrm{p}$-value $=0.003$ ), with 10 years of experience being the cutoff point; providers who had less than 10 years were more likely to quit than those with more years of experience. Among demographic variables, analysis reveals a significant ( $\mathrm{p}$-value=0.001) U-shaped relationship between age and likelihood to quit, whereby the younger (18-25 years) and older (more than 55 years) were more likely to quit than other age groups. Both marital status ( $\mathrm{p}$-value $=0.001)$ and having children $(\mathrm{p}$-value $<0.001)$ were

\section{Table 2 Providers' reported likelihood to quit and} burnout level

\begin{tabular}{|c|c|c|}
\hline Variables & Frequency (N) & Percentage (\%) \\
\hline \multicolumn{3}{|c|}{ Likelihood to quit } \\
\hline Likely & 285 & 39.4 \\
\hline Not sure & 97 & 13.4 \\
\hline Unlikely & 342 & 47.2 \\
\hline \multicolumn{3}{|c|}{ Burnout Level } \\
\hline \multicolumn{3}{|c|}{ Emotional Exhaustion (EE) } \\
\hline High & 165 & 23.2 \\
\hline Medium & 126 & 17.7 \\
\hline Low & 421 & 59.1 \\
\hline \multicolumn{3}{|c|}{ Depersonalization (DP) } \\
\hline High & 98 & 13.8 \\
\hline Medium & 110 & 15.5 \\
\hline Low & 502 & 70.7 \\
\hline \multicolumn{3}{|c|}{ Personal Accomplishment (PA) } \\
\hline High & 132 & 18.7 \\
\hline Medium & 116 & 16.4 \\
\hline Low & 459 & 64.9 \\
\hline
\end{tabular}

correlated with likelihood to quit, with married providers and those with children being less likely to quit.

In terms of work position, likelihood to quit was highest among less specialized providers, including allied health professionals (technicians and assistants), as well as "other health professionals", with proportions of $45.9 \%$ and $48.1 \%$, respectively. Nurses expressed the lowest likelihood to quit their job (36.2\%, p-value=0.025). Moreover, likelihood to quit was significantly associated with the number of years spent in the current PHC center of employment ( $\mathrm{p}$-value $=0.003)$, with providers having worked 'six months to one year' being more likely to quit that those who worked for more years.

Sense of belonging $(\mathrm{p}$-value $=0.023)$ showed to be a significant predictor of likelihood to quit among $\mathrm{PHC}$ providers. Moreover, one-way travel time from place of residence to the $\mathrm{PHC}$ center was also significantly associated with likelihood to quit ( $\mathrm{p}$-value $=0.015$ ); the difference was especially noted at 15 minutes, whereby those who traveled less than 15 minutes were less likely to quit than the rest.

\section{Multinomial logistic regression}

A multinomial logistic regression was conducted to examine the factors significantly associated with likelihood to quit among the PHC providers (Table 4). The goodnessof-fit statistics (Pearson and Deviance) were not significant, suggesting that the data fits the presented model. Two sub-scales of burnout were significantly associated with likelihood to quit: EE and PA. Those with high level of burnout on the EE subscale had 3.46 times the odds of quitting compared to respondents with low level of EE (95\% CI $=2.00-5.99$; p-value $<0.001)$. Those with a moderate level of burnout on the EE subscale had 1.72 times the odds of quitting compared to those with low level of burnout (95\% CI = 0.93-3.15; p-value 0.082).

Respondents with high level of burnout on the PA subscale had 3.05 times the odds of quitting (95\% CI $=1.67$ 5.56; p-value $<0.001$ ) and 2.42 times the odds of being unsure whether to stay or quit $(95 \% \mathrm{CI}=1.06-5.48$; p-value 0.035) as compared to respondents with low level PA. With regards to education, respondents who had a university degree had 2.48 times the odds of quitting compared to those with a medical specialty $(95 \% \mathrm{CI}=1.02-6.08$; p-value 0.046).

Regarding the number of work hours per week, those who worked 1 to 10 hours had 3.15 times the odds of being unsure of their likelihood to quit as compared to those who worked more than 30 hours (95\% CI = 1.22-8.15; p-value 0.018). A couple of findings did not reach the level of significance but are worth noting: Increases in the number of work hours per week was associated with reduced likelihood to quit and full-time employment status served as a protective factor in terms of likelihood to quit. 


\begin{tabular}{|c|c|c|c|c|c|c|c|c|c|}
\hline & & & \multicolumn{6}{|c|}{ Likelihood to quit } & \multirow{3}{*}{$\begin{array}{c}\text { P- } \\
\text { - value }\end{array}$} \\
\hline & & & \multicolumn{2}{|c|}{ Likely } & \multicolumn{2}{|c|}{ Not sure } & \multicolumn{2}{|c|}{ Unlikely } & \\
\hline & & & $\mathrm{N}$ & $\%$ & $\mathrm{~N}$ & $\%$ & $\mathrm{~N}$ & $\%$ & \\
\hline \multirow[t]{9}{*}{ Burnout } & \multirow[t]{3}{*}{$\mathrm{EE}$} & Low & 136 & $32.5 \%$ & 58 & $13.9 \%$ & 224 & $53.6 \%$ & \multirow[t]{3}{*}{$<0.001$} \\
\hline & & Moderate & 50 & $40.3 \%$ & 19 & $15.3 \%$ & 55 & $44.4 \%$ & \\
\hline & & High & 94 & $57.0 \%$ & 18 & $10.9 \%$ & 53 & $32.1 \%$ & \\
\hline & \multirow[t]{3}{*}{ PA } & Low & 159 & $34.9 \%$ & 62 & $13.6 \%$ & 234 & $51.4 \%$ & \multirow[t]{3}{*}{0.010} \\
\hline & & Moderate & 57 & $49.1 \%$ & 16 & $13.8 \%$ & 43 & $37.1 \%$ & \\
\hline & & High & 63 & $47.7 \%$ & 16 & $12.1 \%$ & 53 & $40.2 \%$ & \\
\hline & \multirow[t]{3}{*}{ DP } & Low & 175 & $35.2 \%$ & 72 & $14.5 \%$ & 250 & $50.3 \%$ & \multirow[t]{3}{*}{0.002} \\
\hline & & Moderate & 50 & $45.5 \%$ & 13 & $11.8 \%$ & 47 & $42.7 \%$ & \\
\hline & & High & 55 & $56.1 \%$ & 9 & $9.2 \%$ & 34 & $34.7 \%$ & \\
\hline \multirow[t]{13}{*}{ Training/Education } & \multirow[t]{5}{*}{ Education } & $\begin{array}{l}\text { High school or lower /vocational/ } \\
\text { diploma }\end{array}$ & 45 & $42.1 \%$ & 13 & $12.1 \%$ & 49 & $45.8 \%$ & \multirow[t]{5}{*}{0.045} \\
\hline & & Nursing degree & 71 & $34.3 \%$ & 24 & $11.6 \%$ & 112 & $54.1 \%$ & \\
\hline & & University degree & 55 & $52.4 \%$ & 16 & $15.2 \%$ & 34 & $32.4 \%$ & \\
\hline & & Medical degree & 44 & $35.5 \%$ & 21 & $16.9 \%$ & 59 & $47.6 \%$ & \\
\hline & & Medical Specialty & 68 & $38.2 \%$ & 23 & $12.9 \%$ & 87 & $48.9 \%$ & \\
\hline & \multirow[t]{4}{*}{ Number of years since graduation } & 10 years or less & 142 & $44.8 \%$ & 55 & $17.4 \%$ & 120 & $37.9 \%$ & \multirow{4}{*}{$<0.001$} \\
\hline & & $11-20$ years & 75 & $40.5 \%$ & 22 & $11.9 \%$ & 88 & $47.6 \%$ & \\
\hline & & 21-30 years & 48 & $32.4 \%$ & 14 & $9.5 \%$ & 86 & $58.1 \%$ & \\
\hline & & More than 30 years & 12 & $21.4 \%$ & 5 & $8.9 \%$ & 39 & $69.6 \%$ & \\
\hline & \multirow{4}{*}{$\begin{array}{l}\text { Number of years working in primary } \\
\text { care centers }\end{array}$} & Six months to 1 year & 41 & $45.1 \%$ & 16 & $17.6 \%$ & 34 & $37.4 \%$ & \multirow[t]{4}{*}{0.003} \\
\hline & & $1-5$ years & 113 & $43.1 \%$ & 39 & $14.9 \%$ & 110 & $42.0 \%$ & \\
\hline & & $6-10$ years & 69 & $41.6 \%$ & 21 & $12.7 \%$ & 76 & $45.8 \%$ & \\
\hline & & More than 10 years & 61 & $29.9 \%$ & 21 & $10.3 \%$ & 122 & $59.8 \%$ & \\
\hline \multirow{10}{*}{$\begin{array}{l}\text { Socio-economic } \\
\text { variables }\end{array}$} & \multirow[t]{5}{*}{ Age } & $18-25$ & 40 & $48.8 \%$ & 15 & $18.3 \%$ & 27 & $32.9 \%$ & \multirow[t]{5}{*}{0.001} \\
\hline & & $26-35$ & 97 & $44.3 \%$ & 32 & $14.6 \%$ & 90 & $41.1 \%$ & \\
\hline & & $36-45$ & 86 & $43.2 \%$ & 24 & $12.1 \%$ & 89 & $44.7 \%$ & \\
\hline & & $46-55$ & 49 & $27.7 \%$ & 20 & $11.3 \%$ & 108 & $61.0 \%$ & \\
\hline & & $>55$ & 13 & $31.0 \%$ & 4 & $9.5 \%$ & 25 & $59.5 \%$ & \\
\hline & Marital status & Single & 98 & $45.6 \%$ & 40 & $18.6 \%$ & 77 & $35.8 \%$ & 0.001 \\
\hline & & Married & 174 & $36.9 \%$ & 51 & $10.8 \%$ & 247 & $52.3 \%$ & \\
\hline & & Other & 11 & $31.4 \%$ & 6 & $17.1 \%$ & 18 & $51.4 \%$ & \\
\hline & Children & No & 135 & $44.6 \%$ & 54 & $17.8 \%$ & 114 & $37.6 \%$ & $<0.001$ \\
\hline & & Yes & 150 & $35.6 \%$ & 43 & $10.2 \%$ & 228 & $54.2 \%$ & \\
\hline Work-related & Current position in $\mathrm{PHC}$ & Generalist & 59 & $36.4 \%$ & 25 & $15.4 \%$ & 78 & $48.1 \%$ & 0.025 \\
\hline & & Medical specialist & 61 & $39.6 \%$ & 22 & $14.3 \%$ & 71 & $46.1 \%$ & \\
\hline & & Nurse & 88 & $36.2 \%$ & 29 & $11.9 \%$ & 126 & $51.9 \%$ & \\
\hline & & Allied health professionals & 51 & $45.9 \%$ & 8 & $7.2 \%$ & 52 & $46.8 \%$ & \\
\hline & & Other professional & 26 & $48.1 \%$ & 13 & $24.1 \%$ & 15 & $27.8 \%$ & \\
\hline & Number of years working in this PHC & Six months to 1 year & 58 & $43.6 \%$ & 23 & $17.3 \%$ & 52 & $39.1 \%$ & 0.003 \\
\hline & & $1-5$ years & 136 & $42.1 \%$ & 48 & $14.9 \%$ & 139 & $43.0 \%$ & \\
\hline & & $6-10$ years & 52 & $37.1 \%$ & 14 & $10.0 \%$ & 74 & $52.9 \%$ & \\
\hline & & More than 10 years & 33 & $28.7 \%$ & 10 & $8.7 \%$ & 72 & $62.6 \%$ & \\
\hline & Number of work hours per week & $1-10$ hours & 71 & $41.5 \%$ & 31 & $18.1 \%$ & 69 & $40.4 \%$ & 0.013 \\
\hline
\end{tabular}


Table 3 Association between likelihood to quit and providers' characteristics $(\mathbf{N}=\mathbf{7 5 5})$ (Continued)

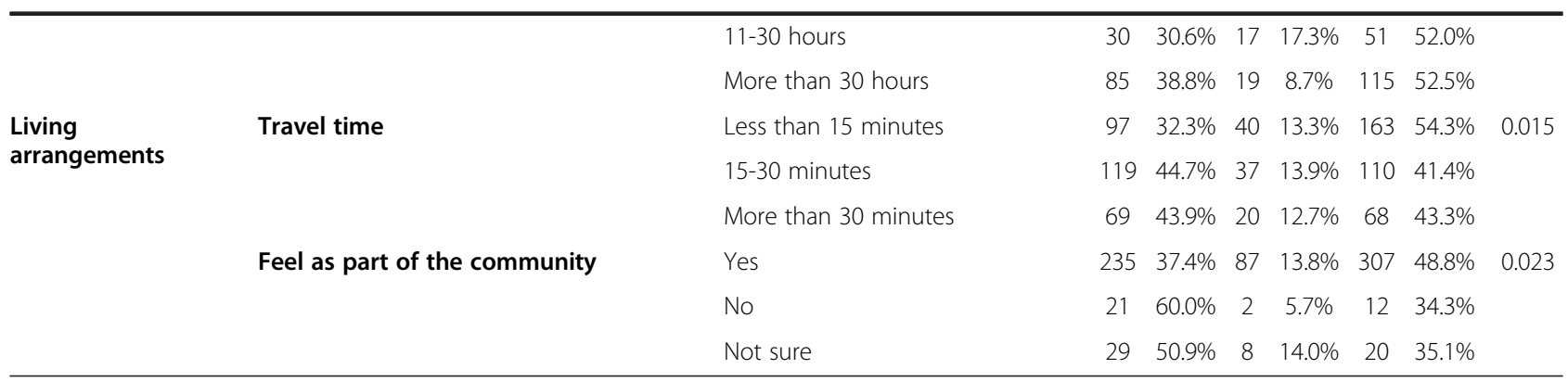

As for number of years working in PHC, providers who had worked 'Six months to one year' had 3.88 times the odds of quitting as compared to those who had worked more than 10 years ( $95 \% \mathrm{CI}=1.36-11.10$; p-value 0.011 ). Moreover, those who had worked 6 to 10 years had 1.99 times the odds of being likely to quit as compared to those who had worked more than 10 years at PHC centers $(95 \%$ $\mathrm{CI}=1.03$-3.84; p-value 0.041).

\section{Discussion}

This study is the first of its kind to systematically investigate factors significantly affecting health providers' retention at PHCs in Lebanon and one of the rare regional attempts to examine this important issue. Results suggest that two of every five PHC providers expressed likelihood to quit their job and an additional $13.4 \%$ are undetermined whether to stay or leave. Such a finding is problematic considering the existing PHC HHR retention crisis observed worldwide and the ongoing attrition of health providers from low and middle income countries to high income countries [11,33-35]; this is of specific relevance to Lebanon as well considering the migration trend of HHR to Gulf countries [36]. Furthermore, it is indicative of a volatile workforce and flags a policy priority to investigate and act upon the underlying causes behind health providers' expressed likelihood to quit their jobs.

Table 4 Regression model testing for predictors of likelihood to quit

\begin{tabular}{|c|c|c|c|c|c|c|c|c|}
\hline & \multicolumn{8}{|c|}{ Likelihood to Quit** } \\
\hline & \multicolumn{4}{|c|}{ Likely vs. Not likely } & \multicolumn{4}{|c|}{ Not sure vs. Not likely } \\
\hline & \multirow[t]{2}{*}{ OR } & \multicolumn{2}{|c|}{$\mathrm{Cl}$} & \multirow[t]{2}{*}{ P-value } & \multirow[t]{2}{*}{ OR } & \multicolumn{2}{|c|}{$\mathrm{Cl}$} & \multirow[t]{2}{*}{ P-value } \\
\hline & & Lower & Upper & & & Lower & Upper & \\
\hline \multicolumn{9}{|l|}{ EE level } \\
\hline High vs. low & 3.46 & 2.00 & 5.99 & $<0.001$ & 0.78 & 0.31 & 1.96 & 0.596 \\
\hline Moderate vs. low & 1.72 & 0.93 & 3.15 & 0.082 & 1.00 & 0.42 & 2.35 & 0.997 \\
\hline \multicolumn{9}{|l|}{ PA level } \\
\hline High vs. low & 3.05 & 1.67 & 5.56 & $<0.001$ & 2.42 & 1.06 & 5.48 & 0.035 \\
\hline Moderate vs. low & 1.78 & 0.98 & 3.23 & 0.060 & 0.97 & 0.40 & 2.36 & 0.944 \\
\hline \multicolumn{9}{|l|}{ Education } \\
\hline Intermediate/high school/vocational or diploma vs. Medical specialty & 1.63 & 0.71 & 3.78 & 0.253 & 2.27 & 0.74 & 6.98 & 0.153 \\
\hline Nursing degree vs. Medical specialty & 0.84 & 0.36 & 1.94 & 0.681 & 1.03 & 0.31 & 3.47 & 0.962 \\
\hline University degree vs. Medical specialty & 2.48 & 1.02 & 6.08 & 0.046 & 2.34 & 0.70 & 7.82 & 0.169 \\
\hline Medical degree vs. Medical specialty & 1.12 & 0.61 & 2.06 & 0.712 & 1.89 & 0.88 & 4.05 & 0.101 \\
\hline \multicolumn{9}{|l|}{ Number of work hours per week } \\
\hline 1 to 10 hours vs. 31 and up hours & 1.83 & 0.92 & 3.64 & 0.083 & 3.15 & 1.22 & 8.15 & 0.018 \\
\hline 11 to 30 hours vs. 31 and up hours & 0.82 & 0.42 & 1.62 & 0.568 & 2.00 & 0.81 & 4.94 & 0.135 \\
\hline \multicolumn{9}{|l|}{ Number of years working in PHC } \\
\hline Six months to 1 year vs. More than 10 years & 3.88 & 1.36 & 11.10 & 0.011 & 3.13 & 0.82 & 11.94 & 0.095 \\
\hline 1 to 5 years vs. More than 10 years & 1.60 & 0.81 & 3.15 & 0.179 & 1.44 & 0.58 & 3.60 & 0.437 \\
\hline 6 to 10 years vs. More than 10 years & 1.99 & 1.03 & 3.84 & 0.041 & 1.21 & 0.47 & 3.09 & 0.690 \\
\hline
\end{tabular}

*P-values below 0.05 are significant and therefore in bold font.

** Goodness-of-fit: Pearson's (chi-square $=7325.0, \mathrm{df}=676, \mathrm{p}$-value $=0.057$ ) and Deviance (chi-square $=645.8, \mathrm{df}=676, \mathrm{p}-\mathrm{value}=0.793$ ). 
One in four PHC providers suffers from a high level of burnout related to EE and one in seven suffers from a high level of burnout related to DP. The observed level of burnout among PHC providers is worrisome for two reasons. First, PHC settings are anticipated to place a lower degree of stress on HHR as compared to the high stress environment of acute hospital care, in which patients are of higher acuity level [37]; however, our findings indicate a relatively elevated degree of stress. Second, there exists a significant and strong association between professional burnout and likelihood to quit. Literature extensively establishes the mediating role of burnout when investigating healthcare providers' turnover within the hospital and PHC setting, as the level of burnout presents as a predictor of job satisfaction [12,29,38].

The findings do not only reflect the presence of elements in the work culture that are precipitating burnout on PHC providers, but also signal a priority issue for $\mathrm{PHC}$ managers to support their staff in order to mitigate the effects of this burnout. Strategies that involve securing adequate staffing, appropriate infrastructure, attractive benefits, supportive work environments, along with professional human resource development practices have shown to allow health workers to respond more effectively to the demands of their jobs, and thus enhance their retention [34,39,40]. Retention strategies must, however, be adapted to meet the needs of HHR working in different settings; a 'one-coatfits-all' approach is doubtful to succeed [39]. Considering the top reasons for quitting that were reported in this study, there should be improvements directed towards remuneration policies. It is recommended that flexibility be built into the current remuneration system at PHC centers, where practices such as but not limited to employment of salary ranges may attract health providers to understaffed and/or underserved areas. More importantly, however, is the pressing need for increased attention towards enhancing education and professional development and boosting the sense of job stability and security amongst providers, as a means for improving role satisfaction within the PHC centers. This recommendation is consistent with literature which has highlighted the comparable importance of nonfinancial incentives to financial incentives in influencing health workers' decisions to remain in the job [41].

Furthermore, stakeholders within the PHC setting are also invited to reflect on the observed U-shaped pattern between age and likelihood to quit. While it is anticipated to observe a higher likelihood to quit amongst older providers aged more than 55 years as they are approaching the age of retirement, it is alarming to observe a higher likelihood of quitting amongst younger providers aged 18-25 years. Although this age group includes new graduates and young professionals who tend to have a high degree of mobility [42], they are the ones with greatest potential to establish, through ap- propriate support and mentorship, lifelong careers in PHC. Efforts to provide safe and conducive work environments that facilitate the integration of new recruits may be wielded through support programs that ease transition into the new work culture. Developed models to attract and retain newly qualified nurses in primary care entail provision of support during the first months of employment, through preceptors and clinical induction programs $[14,43,44]$. Such initiatives have proven their effectiveness in the hospital sector, for example, where they were associated with improvements in selfconfidence in patient care provision, positive results in new nurses' competency and more importantly reduced turnover and increased retention rates $[45,46]$.

The inversely proportional relationship between likelihood to quit amongst health providers and job tenure with the shortest tenure (six months to one year) having the highest likelihood to quit - further supports the need for new staff mentorship and support programs. This is pivotal since almost half of the respondents have been working for a relatively short period of time in the current PHC center of employment and are therefore prone to display a higher likelihood of quitting. This relationship with institutional tenure could be attributed to providers' dissatisfaction with the nature of the job as it compares with their expectations during the early employment period. A study conducted by Stewart et al., showed that the number of years nurses were employed to be significantly related to likelihood to quit; RNs who had been employed less than 2 years in their current positions were 3 times more likely to intend to quit than RNs employed 20 years or more [47]. In another study conducted on nurse migration in Lebanon, El-Jardali et al. found that one out of five registered nurses migrates out of Lebanon within 1-2 years of graduation [36]. On the other hand, the observed decreased likelihood to quit with increasing tenure may be indicative of a sense of organizational commitment amongst those providers- a finding that is supported in literature $[17,48]$.

Another important consideration relates to the educational programs of health providers which may be more focused on preparing them for services in the hospital sector and are potentially making them less acquainted to serving in community settings [49-51]. Moreover, nursing students tend to favor the hospital setting due to a self-perceived lack of clinical experience that drives them to seek support more readily available in a hospital workplace [52]. Studies have shown that changes made to the medical school and residency programs, to better incorporate skills relevant to the PHC setting, have resulted in an increase in the number of providers hired at PHC centers [53]. This underscores the critical role that orientation, mentorship and support programs play in supporting $\mathrm{PHC}$ providers, especially the younger new graduates of them. 
Likelihood to quit is significantly associated with position within the PHC center as the more skilled groups of providers were less likely to quit compared to allied health professionals. A key explanation relates to the fact that allied health professionals receive a relatively lower salary compared to other skilled professionals. Another possibility may revolve around increased job satisfaction stemming from direct contact with patients [54]. Skilled professionals, including physicians and nurses, due to their contact with patients, may acquire an intrinsic sense of reward that might mitigate their likelihoods to quit. This finding exposes a need for managers of PHC centers to dedicate targeted programs to professional with transferrable skills, so as to successfully retain them within their institutions.

Geographical location and sense of belonging to the community are shown to be two significant factors associated with likelihood to quit. More specifically, travel time significantly determined the likelihood of $\mathrm{PHC}$ providers to quit, with 15 minutes of transportation being the cutoff point between being likely and unlikely to quit. This could be explained by the poor public transportation conditions, high cost of gas, and lack of transportation allowances on behalf of the PHC centers in Lebanon. Numerous studies have pointed to geographic location as an important indicator of intention to leave a position within PHC centers and hospitals alike, as it is attributed to a desire for less commute time, as well as community belonging $[15,47,51,53]$. These results are suggestive of a need to staff PHC centers with providers residing in nearby, which may be particularly useful for PHC centers in remote rural areas of Lebanon; employees may be more easily retained with enhanced sense of belonging and easy commute.

This study has a number of shortcomings that warrant mentioning. First, despite the best efforts of the research team to pilot test the survey questionnaire, it cannot be ascertained that all providers were able to understand all the questions; particularly the less educated providers. Second, the study achieved an overall response rate of $60 \%$. Although this response rate falls within the acceptable range for similar studies, the research team is not able to confirm whether the survey respondents are any different than non-respondents. It has to be noted though that most of the non-respondents are those that work on a part-time or casual basis at surveyed PHC centers. Third, despite the presence of some similarities between the private and the public PHC services in Lebanon, the findings of the study are only generalizable to the private PHC sector. Fourth, as a cross-sectional study, it does not allow for examining a causal relationship between the dependent variable of interest, likelihood to quit, and the factors that might affect its presence.

\section{Conclusions}

This study has identified key findings related to the retention of human health resources in primary healthcare centers in Lebanon, a topic that has not been investigated much in the region. Several recommendations can be made to managers at PHC centers with the aim of stabilizing the workforce and ensuring its longevity. Retention strategies should be developed, implemented and evaluated at various centers and for all provider groups, but more importantly for those who appear to be at high risk of quitting. Targeted mentorship and support programs should be dedicated to new graduates and younger recruits who are particularly prone to quitting their jobs.

PHC centers must invest in enhancing the quality of their work environment to reduce stressors in the workplace and improve HHR satisfaction. Globalization and regional competition necessitate the enhancement of HHR compensation and access to professional development opportunities.

The renewed global commitment to PHC as the linchpin of individual and population health does not only necessitate immediate action on the findings of this study but also requires the intervention of multiple stakeholders, including: managers, syndicates and orders, the Ministries of Population Health and Social Affairs, educational and research institutions. The study also paves the way for future studies both locally and regionally. While some of the findings and recommendations may be context specific, others certainly would apply to other countries in the region.

Access to people-centered primary healthcare is widely recognized as an important facilitator of overall population health, it is of pivotal importance that PHC stakeholders exercise all due diligence to foster a work milieu that is conducive to staff retention and would allow for sustainability in provision of health services.

\section{Competing interests}

None to declare for any of the authors.

\section{Authors' contributions}

MA: Conceptualized the study, wrote the proposal, secured funding, lead all aspects of data collection and analysis and prepared the first draft of the manuscript. He approved the final submitted version of this manuscript. Ss: Conceptualized the study, contributed to data analysis of collected information and helped in the write up of the manuscript. He approved the final submitted version of this manuscript. FE: Helped with the conceptualization the study, contributed to the write up of the funded grant and significantly contributed to analysis and write up of the manuscript. He reviewed the manuscript and approved the final submitted version. HD: Lead the statistical analysis carried out in this study and contributed to the write up of the manuscript. He reviewed the manuscript and approved the final submitted version. YM: Contributed to data entry and analysis. Provided valuable support with the referencing and editing of the final version of the manuscript. All authors read and approved the final manuscript.

\section{Acknowledgement}

The authors would like to acknowledge the generous funding for this study by the Qatar National Research Fund, National Priorities Research Program for a study titled 'Investigating the Terrain of Health Human Resources at Primary Health Care Centers in Lebanon and Qatar'. 


\section{Author details}

Assistant Professor, Faculty of Health Sciences, Department of Health Management and Policy, American University of Beirut, Riad El-Solh, Beirut 1107 2020, Lebanon. ${ }^{2}$ Associate Professor, Faculty of Health Sciences, Department of Health Management and Policy, American University of Beirut, Riad El-Solh, Beirut 1107 2020, Lebanon. ${ }^{3}$ Assistant Professor, School of Pharmacy, Lebanese American University, P.O.Box 36, Byblos, Lebanon. ${ }^{4}$ Research Assistant, Faculty of Health Sciences, Department of Health Management and Policy, American University of Beirut, Riad El-Solh, Beirut 1107 2020, Lebanon.

Received: 17 May 2012 Accepted: 30 October 2012

Published: 22 November 2012

\section{References}

1. Labonte R, Sanders D, Baum F, Schaay N, Packer C: Implementation, effectiveness and political context of comprehensive primary health care: preliminary findings of a global literature review. Aust J Prim Health 2008, 14(3):58

2. Biola H, Green LA, Phillips RL, Guirguis-Blake J, Fryer GE: The U.S. primary care physician workforce: minimal growth 1980-1999. Am Fam Physician 2003, 68(8):1483.

3. Kringos DS, Boerma WG, Hutchinson A, van der Zee J, Groenewegen PP: The breadth of primary care: a systematic literature review of its core dimensions. BMC Health Serv Res 2010, 10:65.

4. Starfield B, Shi L, Macinko J: Contribution of primary care to health systems and health. Milbank Q 2005, 83(3):457-502.

5. Dussault $\mathrm{G}$, Dubois CA: Human resources for health policies: a critical component in health policies. Hum Resour Health 2003, 1(1):1.

6. Hurst K: Primary and community care workforce planning and development. J Adv Nurs 2006, 55(6):757-769.

7. Anand S, Barnighausen T: Human resources and health outcomes: crosscountry econometric study. Lancet 2004, 364(9445):1603-1609.

8. Hongoro C, McPake B: How to bridge the gap in human resources for health. Lancet 2004, 364(9443):1451-1456.

9. Jenkins-Clarke S, Carr-Hill R: Changes, challenges and choices for the primary health care workforce: looking to the future. J Adv Nurs 2001, 34(6):842-849.

10. Dubois CA, McKee M, Nolte E: Analysing trends, opportunities and challenges. In Human resources for health in Europe. Edited by Dubois CA, McKee M, Nolte E. Berkshire: Open University Press; 2006:15-40.

11. El-Jardali F, Jamal D, Abdallah A, Kassak K: Human resources for health planning and management in the Eastern Mediterranean region: facts, gaps and forward thinking for research and policy. Hum Resour Health 2007, 5:9.

12. Aiken LH, Clarke SP, Sloane DM, Sochalski J, Silber JH: Hospital nurse staffing and patient mortality, nurse burnout, and job dissatisfaction. JAMA 2002, 288(16):1987-1993.

13. Blount FA, Miller BF: Addressing the workforce crisis in integrated primary care. J Clin Psychol Med Settings 2009, 16(1):113-119.

14. Drennan V, Andrews $S$, Sidhu R, Peacock R: Attracting and retaining nurses in primary care. Br J Community Nurs 2006, 11(6):242-246.

15. Odom Walker K, Ryan G, Ramey R, Nunez FL, Beltran R, Splawn RG, Brown AF: Recruiting and retaining primary care physicians in urban underserved communities: the importance of having a mission to serve. Am J Public Health 2010, 100(11):2168-2175.

16. Nowak $P$, Holmes $G$, Murrow J: A model for reducing health care employee turnover. J Hosp Mark Public Relations 2010, 20(1):14-25.

17. Ingersoll GL, Olsan T, Drew-Cates J, DeVinney BC, Davies J: Nurses' job satisfaction, organizational commitment, and career intent. J Nurs Adm 2002, 32(5):250-263.

18. Shields MA, Ward M: Improving nurse retention in the National Health Service in England: the impact of job satisfaction on intentions to quit. $J$ Health Econ 2001, 20(5):677-701.

19. Cowin $L:$ The effects of nurses' job satisfaction on retention: an Australian perspective. J Nurs Adm 2002, 32(5):283-291.

20. Tourangeau $A E$, Cranley LA: Nurse intention to remain employed: understanding and strengthening determinants. J Adv Nurs 2006, 55(4):497-509.

21. Larrabee JH, Janney MA, Ostrow CL, Withrow ML, Hobbs GR Jr, Burant C: Predicting registered nurse job satisfaction and intent to leave. J Nurs Adm 2003, 33(5):271-283.
22. OToole K, Schoo A, Stagnitti K, Cuss K: Rethinking policies for the retention of allied health professionals in rural areas: a social relations approach. Health Policy 2008, 87(3):326-332.

23. Hayes LJ, O'Brien-Pallas L, Duffield C, Shamian J, Buchan J, Hughes F, Spence Laschinger HK, North N, Stone PW: Nurse turnover: a literature review. Int J Nurs Stud 2006, 43(2):237-263.

24. Srouji E: A rural primary health care center for Lebanon. J Med Liban 1972, 25(6):529-540

25. Ammar W: The Primary Healthcare Network, Health Beyond Politics. Cairo: WHO Eastern Mediterranean Regional Office; 2009.

26. Kronfol NM: Rebuilding of the Lebanese health care system: health sector reforms. East Mediterr Health J 2006, 12(3-4):459-473.

27. WHO: The Work of WHO in the Eastern Mediterranean Region, Annual Report of the Regional Director 2005. Cairo: WHO/EMRO; 2005.

28. Murrells T, Robinson S, Griffiths P: Is satisfaction a direct predictor of nursing turnover? Modelling the relationship between satisfaction, expressed intention and behaviour in a longitudinal cohort study. Hum Resour Health 2008, 6:22.

29. Leiter MP, Maslach C: Nurse turnover: the mediating role of burnout J Nurs Manag 2009, 17(3):331-339.

30. Kivimaki M, Vanhala A, Pentti J, Lansisalmi H, Virtanen M, Elovainio M, Vahtera J: Team climate, intention to leave and turnover among hospital employees: prospective cohort study. BMC Health Serv Res 2007, 7:170

31. Zurmehly J, Martin PA, Fitzpatrick JJ: Registered nurse empowerment and intent to leave current position and/or profession. J Nurs Manag 2009, 17(3):383-391.

32. Maslach C, Jackson S, Leiter M: Maslach Burnout Inventory Manual. Third edth edition. Mountain View (CA): CPP. 1996

33. Lehmann U, Dieleman M, Martineau T: Staffing remote rural areas in middle- and low-income countries: a literature review of attraction and retention. BMC Health Serv Res 2008, 8:19

34. Willis-Shattuck M, Bidwell $P$, Thomas $S$, Wyness $L$, Blaauw D, Ditlopo $P$. Motivation and retention of health workers in developing countries: a systematic review. BMC Health Serv Res 2008, 8:247.

35. Buchan J, Sochalski J: The migration of nurses: trends and policies. Bull World Health Organ 2004, 82(8):587-594.

36. El-Jardali F, Merhi M, Jamal D, Dumit N, Mouro G: Assessment of nurse retention challenges and strategies in Lebanese hospitals: the perspective of nursing directors. J Nurs Manag 2009, 17(4):453-462.

37. Beecroft PC, Dorey F, Wenten M: Turnover intention in new graduate nurses: a multivariate analysis. J Adv Nurs 2008, 62(1):41-52.

38. Linzer M, Manwell LB, Williams ES, Bobula JA, Brown RL, Varkey AB, Man B, McMurray JE, Maguire A, Horner-lbler B, Schwartz MD: MEMO (Minimizing Error, Maximizing Outcome) Investigators: Working conditions in primary care: physician reactions and care quality. Ann Intern Med 2009, 151(1):28-36. W6-9.

39. Buykx P, Humphreys J, Wakerman J, Pashen D: Systematic review of effective retention incentives for health workers in rural and remote areas: towards evidence-based policy. Aust J Rural Health 2010, 18(3):102-109.

40. Lammintakanen J, Kivinen T, Kinnunen J: Managers' perspectives on recruitment and human resource development practices in primary health care. Int I Circumpolar Health 2010, 69(5):462-469.

41. Lagarde M, Blaauw D: A review of the application and contribution of discrete choice experiments to inform human resources policy interventions. Hum Resour Health 2009, 7:62

42. Wieck KL, Dols J, Landrum P: Retention priorities for the intergenerational nurse workforce. Nurs Forum 2010, 45(1):7-17.

43. Schoessler $\mathrm{M}$, Waldo $\mathrm{M}$ : The first 18 months in practice: a developmental transition model for the newly graduated nurse. J Nurses Staff Dev 2006, 22(2):47-52. uiz 53-4.

44. Lavoie-Tremblay M, O'Brien-Pallas L, Gelinas C, Desforges N, Marchionni C: Addressing the turnover issue among new nurses from a generational viewpoint. J Nurs Manag 2008, 16(6):724-733.

45. Park $M$, Jones $C B$ : A retention strategy for newly graduated nurses: an integrative review of orientation programs. J Nurses Staff Dev 2010 26(4):142-149.

46. Newhouse RP, Hoffman JJ, Suflita J, Hairston DP: Evaluating an innovative program to improve new nurse graduate socialization into the acute healthcare setting. Nurs Adm Q 2007, 31(1):50-60.

47. Stewart NJ, D'Arcy C, Kosteniuk J, Andrews ME, Morgan D, Forbes D, Macleod ML, Kulig JC, Pitblado JR: Moving on? Predictors of intent to leave among rural and remote RNs in Canada. J Rural Health 2011, 27(1):103-113. 
48. Wagner $\mathrm{CM}$ : Organizational commitment as a predictor variable in nursing turnover research: literature review. J Adv Nurs 2007, 60(3):235-247.

49. Latter S, Speller V, Westwood G, Latchem S: Education for public health capacity in the nursing workforce: findings from a review of education and practice issues. Nurse Educ Today 2003, 23(3):211-218.

50. Frenk J, Chen L, Bhutta ZA, Cohen J, Crisp N, Evans T, Fineberg H, Garcia P, Ke Y, Kelley P, Kistnasamy B, Meleis A, Naylor D, Pablos-Mendez A, Reddy S, Scrimshaw S, Sepulveda J, Serwadda D, Zurayk H: Health professionals for a new century: transforming education to strengthen health systems in an interdependent world. Lancet 2010, 376(9756):1923-1958.

51. Rabinowitz HK, Diamond JJ, Markham FW, Paynter NP: Critical factors for designing programs to increase the supply and retention of rural primary care physicians. JAMA 2001, 286(9):1041-1048.

52. Heslop L, Mclntyre M, Ives G: Undergraduate student nurses' expectations and their self-reported preparedness for the graduate year role. J Adv Nurs 2001, 36(5):626-634

53. Savageau JA, Ferguson WJ, Bohlke $\mathrm{L}$, Cragin $\sqcup$, , O'Connell E: Recruitment and retention of primary care physicians at community health centers: a survey of Massachusetts physicians. J Health Care Poor Underserved 2011, 22(3):817-835.

54. Grunfeld E, Zitzelsberger L, Coristine M, Whelan TJ, Aspelund F, Evans WK: Job stress and job satisfaction of cancer care workers. Psychooncology 2005, 14(1):61-69.

doi:10.1186/1472-6963-12-419

Cite this article as: Alameddine et al:: The retention of health human resources in primary healthcare centers in Lebanon: a national survey. BMC Health Services Research 2012 12:419.

\section{Submit your next manuscript to BioMed Central and take full advantage of:}

- Convenient online submission

- Thorough peer review

- No space constraints or color figure charges

- Immediate publication on acceptance

- Inclusion in PubMed, CAS, Scopus and Google Scholar

- Research which is freely available for redistribution 